fluorescence at all, while the inside which had cooled more slowly, and was found to be crystalline, gave a very brilliant green glow.

Calcium metaborate can by very sudden cooling be obtained in a vitreous state showing no fluorescence, while the crystalline variety gives a moderate blue glow. The presence of manganese induces no fluorescence in the vitreous form, but a very brilliant green in the crystalline modification. Evidence has been obtained that many substances which, in their crystalline form, fluoresce brightly, exhibit no glow in the amorphous or vitreous state.

From numerous experiments it is concluded that the green fluorescence of X-ray tubes is associated with the presence of a notable quantity of calcium and a relatively small amount of manganese, that a truly vitreous glass exhibits little, if any, fluorescence, and that a glass containing manganese can only be kept in this condition by extremely sudden cooling.

Without suggesting that ordinary $\mathrm{X}$-ray glass has any definitely crystalline structure, the evidence would indicate that something akin to this is more readily obtained when manganese is present.

A tendency to crystallisation may be so slight in a glass as not to interfere with its use under ordinary working conditions, but there is no advantage in fostering this tendency. The presence of manganese in any appreciable amount introduces other defects of minor importance. I mention them only to emphasise the point made before, that if the intensity of green fluorescence seen in many foreign $\mathrm{X}$-ray tubes is considered imperative it can only be obtained by sacrificing some of the good working qualities of the new and purer English glass.

Herbert Jackson.

University of London, King's College, June 23.

\section{The Magnetic Storm and Solar Disturbance of} June 17, 1915.

The magnetic storm described in Nature of June 24 by the Rev. A. L. Cortie seems to have been larger at Stonyhurst than at Kew. The extreme westerly position of the declination needle at Kew occurred about I.30 p.m., and the extreme easterly position about 5.37 p.m., the total range being about $72^{\prime}$. Between 5 and $6 \mathrm{p} . \mathrm{m}$. the movements had a range of $6 \mathrm{I}^{\prime}$. I am not clear which of the two corresponds to the $9 \mathrm{r} \cdot 5^{\prime}$ mentioned by Father Cortie, but either is substantially less, even allowing for the fact that the strength of the horizontal field is about 6 per cent. higher at Kew than at Stonyhurst. This is, of course, quite in accordance with the usual tendency for disturbance to be greater in higher latitudes, but it helps to illustrate the fact that whatever the ultimate source may be, terrestrial position counts for a good deal. The total range shown by the horizontal force at Kew was about $460 \gamma\left(\mathrm{I} \gamma \equiv \mathrm{I} \times \mathrm{IO}^{-5}\right.$ C.G.S.), the maximum occurring about 5.42 p.m., and the minimum about 9.30 a.m.

Father Cortie's remarks on the absence of the rapid oscillations sometimes characteristic of magnetic storms refer, I presume, to the time 4 to $6 \mathrm{p} . \mathrm{m}$., when the largest movements occurred. Earlier in the day, for instance, near 6 a.m., the oscillatory character was fairly prominent at Kew. One would, in fact, have expected to hear of telegraphic interruptions.

Father Cortie seems to associate the magnetic storm with a particular spot or group of spots on the sun. That at least is what one would naturally infer from his remark: "Such a close approximation of the position of the spot and the earth referred to the sun's central meridian during a magnetic storm is very unusual." Whether magnetic storms are directly due to the emission of electrons from the sun, and, if so, whether the emission is localised in sun-spots, are questions on which there is a diversity of opinion. The present case seems a good example of the difficulties in the way of a final decision. Father Cortie tells us that "on June $17-18$ there were no fewer than seven groups of spots visible." On the other hand, the disturbance of June 17 , though much the largest, was by no means the only magnetic disturbance about the time. There was, as Father Cortie mentions, what is usually termed a "sudden commencement" about I.50 a.m.-I make it a minute or two later-on June i 7 , but there was another-somewhat larger-about I p.m. on June I6. The subsequent disturbance on June 16 was not large, and after 6 p.m. the conditions were fairly normal. We should naturally regard the disturbances on June I6 and I7 as distinct. Then on June $2 \mathrm{I}$, about 3 . ro p.m., there was yet another "sudden commencement," the largest of the three, and this was followed by a considerable disturbance lasting to the end of June 22. "Sudden commencements," even when small, are usually recognisable over at least the greater part of the world. Of all types of disturbance they seem to have the best claim to a cosmic origin. The subsequent disturbances, when there are any worth mentioning - which is not always the case-show much more rapid local variation.

We have here, then, three disturbances with sudden commencements in the course of about five days, so that even accepting the sun-spot emission theory, in the absence of special identification marks, the association of one particular disturbance with one particular spot or group of spots would seem to be arbitrary.

Another aspect of the case is that magnetic disturbances sometimes occur when sun-spots are conspicuously few or wholly absent. For instance, there was a "sudden commencement" of considerable size on June 7, at a time when Father Cortie tells us the sun was almost free of spots.

The fact that magnetic disturbances occur at intervals of from twenty-six to twenty-eight days more frequently than is accountable for by pure chance is obviously consistent with the sun-spot emission theory; but it does not necessarily favour it. Quiet magnetic conditions show the "twenty-seven-day" period to practically the same extent as disturbed conditions. Sometimes disturbance is the rule, and quiet conditions the exception, and it is not clear that the one phenomenon is more fundamental than the other. There seems a difficulty in associating quiet conditions with some limited area, some "anti-spot," on the sun.

June 26 .

\section{The Names of Physical Units.}

MaY I point out that Dr. Guillaume is wrong in suggesting, in his letter in NaTURE of June 17 , that the adjective "specific," employed in connection with physical magnitudes, has no constant and definite meaning? "Specific" is the adjective of "species," and the "specific resistance of iron" is that function of the resistance of a piece of iron and the other physical magnitudes characteristic of the piece which is the same for all pieces which belong to the species "iron." The statement made in the last sentence is true if for "resistance" be substituted any other magnitude to which "specific" is attached, and for "iron" any other form of matter which is recognised as a "species."

I am not urging that the retention of the term "specific" is desirable; on that matter I offer no opinion. I am only urging that the word has a perfectly definite and constant meaning.

Teddington, June 22 . NORMaN R. Campbell.

NO. 2383 , VOL. 95] 